\title{
Impacto familiar en la recuperación de personas adultas críticamente enfermas: una revisión de la literatura
}

\author{
Family impact on the recovery of critically ill adults: a review of the \\ literature
}

\section{Impacto familiar na recuperação do adulto criticamente enfermo: uma revisão de literatura}

\author{
Paula Ceballos-Vásquez ${ }^{1 *}$ \\ (D) https://orcid.org/0000-0002-3804-5146 \\ María Fernanda Campos-Fuentes ${ }^{2}$ \\ (iD) https://orcid.org/0000-0002-3101-7070 \\ Valentina González-Alegría ${ }^{3}$ \\ iD https://orcid.org/0000-0001-6379-9030 \\ Camila Lobos-Lavín ${ }^{4}$ \\ (iD) https://orcid.org/0000-0003-1128-462X
}

1. Doctora en Enfermería. Departamento de Enfermería. Universidad Católica del Maule.

2. Enfermera Clínica. Especialista en Cuidados Críticos del Adulto de la Universidad Católica del Maule.

3. Enfermera Clínica. Especialista en Cuidados Críticos del Adulto de la Universidad Católica del Maule.

4. Enfermera Clínica. Especialista en Cuidados Críticos del Adulto de la Universidad Católica del Maule.

*Autor para correspondencia: pceballos@ucm.cl

Recibido: $02 / 05 / 2020$

Aceptado: $08 / 03 / 2021$

\section{Resumen}

Introducción: Las unidades de paciente crítico son descritas por las familias de las personas hospitalizadas como un lugar apartado, con acceso restringido, donde la gravedad y complejidad del usuario es lo primordial. A pesar de contar con evidencia de lo importante que podría ser considerar e incluir a los familiares en la recuperación de las personas críticamente enfermas ha sido subvalorada y difícil de concretar por las condiciones complejas de 
estas unidades. Objetivo: Identificar en la literatura el impacto que tiene la familia en la recuperación de las personas adultas hospitalizadas en las unidades de cuidados intensivos. Metodología: Revisión de la literatura, en las bases de datos CINAHL Complete, Scopus, PubMed y Scielo, en periodo de búsqueda del 2014 al 2019. Resultados: Se analizaron 9 artículos y en base a lectura crítica se seleccionaron los aspectos más relevantes para su estudio. Respecto a la distribución por años el 33,3\% de los artículos fue publicado el año 2016. Respecto a la distribución geográfica, el 44,4\% de los manuscritos seleccionados fueron realizados en Europa. Conclusión: Existe una brecha investigativa que relacione el impacto de las familias en la recuperación de los usuarios. Los estudios localizados evidencian datos con tendencias positivas, sin embargo, estos emergen de forma indirecta. Por ello, se propone realizar estudios que entreguen estrategias innovadoras con resultados concretos para verificar dichos beneficios, los cuales sean replicables en otras unidades críticas.

Palabras clave: Rehabilitación; Paciente; Familia; Cuidados críticos (DeCs).

\begin{abstract}
Introduction: Critical care units are described by the families of hospitalized individuals as a secluded place, with restricted access, where the severity and complexity of the patient is paramount. Despite evidence of how important it could be to consider and include family members in the recovery of critically ill persons, this has been undervalued and is difficult to achieve due to the complex conditions of these units. Objective: To identify in the literature the impact that the family has on the recovery of hospitalized adults in intensive care units. Methodology: Literature review in the CINAHL Complete, Scopus, PubMed and Scielo databases, by searching for the 2014 to 2019 period of time. Results: 9 articles were analyzed and based on critical reading the most relevant aspects were selected for study. Regarding the distribution by years, $33.3 \%$ of the articles were published in 2016. Regarding geographical distribution, $44.4 \%$ of the selected manuscripts were written in Europe. Conclusion: There is a research gap that relates the impact of the family on the recovery of the patient. The identified studies show data with positive trends. However, these trends emerge in an indirect way. Therefore, it is proposed to carry out studies that provide innovative strategies with concrete results to verify these benefits, which can be applied in other intensive care units.
\end{abstract}

Key words: Rehabilitation; Patient; Family; Critical Care (DeCs).

\begin{abstract}
Abstrato
Introdução: As unidades dos pacientes críticos são descritas, pelas famílias, dos hospitalizados como um lugar separado, com acesso restrito, em que a gravidade e a complexidade do usuário é prioridade. Embora, exista evidências que poderia ser importante incluir os familiares na recuperação dessas pessoas, criticamente enfermas, isso é subvalorizado e difícil de acontecer, devido as condições complexas dessas unidades. Objetivo: Identificar o impacto na literatura que tem a familia na recuperação das pessoas adultas hospitalizadas em unidades de cuidado intensivo. Métodos: Revisão de literatura nas bases de dados CINAHL completo, Scopus, Pubmed e Scielo. O período da busca foi de 2014 a 2019. Resultados: Foi analisado 9 artigos, os aspectos mais relevantes dos estudos foram descritos. Em relação a distribuição temporal, 33,3\% foram publicados no ano de 2016. Quanto a distribuição geográfica, $44 \%$ das produções foram realizadas na Europa. Conclusão: Existe uma lacuna de estudos que relacione o impacto que as famílias causam na recuperação, os dados favoráveis se obteve de
\end{abstract}


maneira indireta aos estudos encontrados. Se propõe realizar estudos que forneçam estratégias inovadoras, com resultados concretos para verificar os beneficios e que sejam aplicáveis em todas unidades de tratamento crítico.

Palavras-chave: Reabilitação; Paciente; Família; Cuidados Intensivos (DeCs).

\section{Introducción}

Las unidades de cuidados críticos son descritas por las familias de los usuarios internados en ellas, como un lugar frío y lejano, con acceso restringido y exclusivo, donde la gravedad y la complejidad de la persona enferma es lo primordial, motivo por el cual se excluye a la familia y entorno, con la idea de otorgar una mejor atención clínica a la persona críticamente enferma ${ }^{(1-3)}$. Autores indican que las unidades de cuidados intensivos se distinguen de otras áreas hospitalarias por una alta proporción de personal por paciente y acceso a equipos tecnológicos avanzados que no están disponibles de forma rutinaria en ningún otro lugar del hospital ${ }^{(4)}$. Por lo expuesto, la complejidad de dichas unidades pudiera generar objeciones respecto al acceso libre de las familias, sin embargo, extender las horas de visita en unidades de cuidados intensivos y la participación de la familia en la planificación de los cuidados de la persona críticamente enferma, han sido recomendados por sociedades internacionales y organizaciones sanitarias. Así como lo expone el Comité Nacional Italiano de Bioética (INCB), quien destacó el hecho de liberalizar las políticas de visitas es una expresión concreta del principio de respeto por la persona, y es consistente con los principios de autonomía, beneficencia y no maleficencia ${ }^{(5)}$. A la vista de la INCB, basado en el conocimiento científico, la presencia de seres queridos al lado de la cama de ninguna manera constituye una amenaza para el paciente. Por el contrario, tiene un impacto beneficioso tanto en el usuario como en la familia. En particular, la INCB declara que "desde el punto de vista ético es injustificable; a menos que en casos absolutamente excepcionales; no realizar una acción positiva que pueda proporcionar beneficios para el paciente". Solo por motivos éticos y clínicos de riesgos graves para la salud pueden justificar la restricción de visitas ${ }^{(5)}$.

A pesar de estas recomendaciones y la evidencia científica, las horas de visita siguen siendo restringidas en la mayoría de las unidades de cuidados intensivos de muchos países ${ }^{(6)}$. Estudios revelan la 
importancia de involucrar a las familias en el marco de rehabilitación con el propósito de optimizar los resultados. Entre los beneficios probables incluyen redirigir la angustia psicológica familiar a un rol participativo activo, humanizar la enfermedad del paciente y la experiencia de recuperación, apoyando al personal y al sistema de atención médica más allá de las limitaciones del tiempo de terapia ${ }^{(7)}$.

Dada la complejidad de las unidades de cuidados intensivos y la necesidad latente de mejorar la salud de las personas críticamente enfermas, se pretende identificar y evidenciar el impacto que tiene la familia en la recuperación de las personas enfermas dentro de estas unidades. Sustentando el positivismo de dicha actividad, se podrán proponer futuros lineamientos y evidencia teórica sostenible que permita establecerla como una herramienta estratégica para brindar un cuidado holístico.

\section{Objetivo}

Identificar en la literatura el impacto que tiene la familia en la rehabilitación y recuperación de las personas adultas hospitalizadas en unidades de cuidados intensivos.

\section{Metodología}

Se realizó una revisión de la literatura para responder a la pregunta ¿Cuál será el impacto que genera la familia en la rehabilitación de las personas adultas hospitalizadas en la unidad de cuidados críticos?, para estructurar la pregunta se utilizó estrategia PICOT ${ }^{(8)}$. Para la revisión, se utilizaron las bases de datos CINAHL Complete, Scopus, PubMed y Scielo con 4 descriptores consultados español e inglés en los Descriptores en Ciencias de la Salud (DeCS) y Medical Subject Headings (MeSH). En primer lugar, se seleccionaron los

descriptores que se detallan en la Tabla 1. Se puntualiza que Rehabilitation se usó como sinónimo de Recovery, para una búsqueda más amplia. 
Tabla 1. Descriptores DeCs y MeSH

\begin{tabular}{llll}
\hline Descriptores & MeSH & Descriptores & DeCs \\
\hline Rehabilitation & Indexado & Rehabilitación & Indexado \\
Patient & Indexado & Paciente & Indexado \\
Family & Indexado & Familia & Indexado \\
Critical Care & Indexado & Cuidados Críticos & Indexado \\
\hline
\end{tabular}

Fuente: Elaboración propia.

Se continuó ingresando a las bases de datos anteriormente mencionadas con el buscador booleano AND, incluyendo las 4 palabras claves en el siguiente orden: rehabilitation AND patient AND family AND critical care, con el fin de hacer una búsqueda general. Se indica que la búsqueda se limitó por los siguientes Ítems: publicados entre los años 2014 - 2019, investigaciones originales, categoría adulta y nursing y de exclusión: categoría pediátrica. Las etapas de este manuscrito fueron: planteamiento de la pregunta, búsqueda en la literatura de la información a partir de buscadores y bases de datos electrónicas, análisis de la literatura, redacción de los resultados. Para la recolección de los datos se utilizó un instrumento de elaboración propia que incluyó los siguientes Ítems: identificación de los autores, título del artículo, objetivos del manuscrito y resultados o conclusiones relevantes para la revisión y conclusiones.

Así, en CINAHL Complete se obtuvieron un total de 46 artículos, Scopus 353 artículos, PubMed con 632 artículos y finalmente en Scielo 13 artículos. En base a esto se comenzó a limitar las búsquedas a través de los criterios de selección (inclusión y exclusión) y cambio del orden de las palabras claves. Se seleccionaron 4 artículos de CINAHL Complete, 7 artículos de PubMed, 5 artículos SCOPUS y 2 de Scielo. De los 18 artículos ya seleccionados fueron íntegramente leídos en la búsqueda de obtener información respecto del objetivo de la revisión, en donde se realizó un análisis crítico y confirmación de los criterios de inclusión y exclusión por lo que seleccionaron para esta revisión 9 artículos.

\section{Resultados}

Respecto a la distribución por años de los artículos seleccionados, se destaca que el 33,3\% de ellos fue publicado el año 2016 y un 22,2\% el 2019. Respecto a la distribución geográfica, el $44,4 \%$ de los manuscritos 
seleccionados fueron realizados en Europa, mientras que un 33,3\% de los artículos fueron desarrollados en Australia y solo un $11,1 \%$ fue desarrollado en Latinoamérica. La tabla 2, detalla cada artículo seleccionado para esta revisión, en orden cronológico de publicación, lo que permitió establecer una lectura comparativa entre ellos, contrastando objetivos y conclusiones obtenidos por cada autor.

\section{Discusión}

Esta revisión de la literatura emerge con el objetivo de identificar el impacto que tiene la familia en la rehabilitación y recuperación de las personas adultas hospitalizadas en unidades de cuidados intensivos. En dicha recuperación están involucrados aspectos físicos, psicológicos y experiencias emocionales, tanto de la familia de la persona críticamente enferma como del equipo de salud, en estas interacciones se evidencian ventajas y desventajas, limitaciones, al igual que la relación con la rehabilitación durante la hospitalización como también el alta en la unidad de cuidados intensivos.

Como primer aspecto, se destaca la importancia de fortalecer la investigación en la temática, con la finalidad de comprender mejor a las familias ${ }^{(4,7,15)}$. De esta manera ellas, pueden modificar conducta y aportar a la mejora del usuario críticamente enfermo. Ya que es importante saber o conocer como los enfermeros clínicos de las unidades de cuidados intensivos pueden promover un ambiente centrado en la familia, incluyéndola en las actividades de rehabilitación donde corresponda. Los estudios revisados postulan que, para empoderar a las familias en el marco de rehabilitación, se necesita estar abierto y dispuesto a aprender de y con los usuarios y sus familias y comprender que todas estas acciones tienen el potencial de conducir a un mejor resultado ${ }^{(9,14)}$. Se recomienda investigación futura para explorar esta área más a fondo ${ }^{(7,10)}$. Las autoras postulan que sería interesante y muy necesario desarrollar otros estudios con diversos abordajes, es decir cuantitativos, cualitativos y mixto, que permitan responder a las brechas de conocimiento respecto al impacto que genera la familia en la recuperación de los usuarios con alteraciones críticas de salud para responder a preguntas como: ¿existen beneficios macrohemodinámicos en los usuarios con apoyo familiar en la hospitalización? ¿Cuál es el tiempo que se necesita para su implementación 
el apoyo familiar en UCI? ¿Cuántos recursos se necesitan para establecer estrategias sólidas respecto a la incorporación familiar en la recuperación de las personas críticamente enfermas? ¿Deberían desarrollarse, desde el equipo de salud, nuevos protocolos de visitas a las unidades críticas en Chile? ¿Desarrollar más estudios permitirá derribar las barreras visualizadas en este estudio y vivenciadas en lo asistencial?

Tabla 2. Resumen de artículos seleccionados para la realización de revisión narrativa posterior a la aplicación de criterios de inclusión y lectura crítica $(n=9)$.

\section{Nombre del estudio/ \\ Lugar y año de publicación}

\section{Objetivos}

Conclusión y/o resultados
E.1 From spouse to caregiver and back: a grounded theory study of postintensive care unit spousal caregiving/

Dinamarca, $2015^{(9)}$.
Explorar los desafíos y las actividades de cuidado que deben realizar y enfrentar los cónyuges durante el primer año de recuperación de los pacientes hospitalizados en las unidades de cuidados intensivos.
El cuidador informal (cónyuge) se dirige a cubrir las principales preocupaciones y necesidades de su ser querido para promover su recuperación y al mismo tiempo mantener vida de pareja y social. El estudio evidencia que el cuidador se involucra en un proceso dinámico que consta de 4 elementos: comprometerse con el cuidado del paciente, adquirir habilidades de cuidado, negociar el nivel de cuidado y abandonar gradualmente rol de cuidador en el proceso avanzado de la recuperación. Se destaca el apoyo vital proporcionado por los cuidadores informales y su papel activo en todo el proceso de recuperación. Sin embargo, es necesario brindar apoyo a los familiares y cónyuges posterior al alta por parte de los equipos de salud. Se alienta a los profesionales sanitarios, ya sea intra y extrahospitalarios a ser partícipes en proporcionar información y apoyo desde al ingreso del usuario hasta su recuperación posterior al alta.

E.2 Visita restrictiva/visita no Evidenciar el impacto restrictiva en una unidad de la visitas restrictivas de paciente crítico adulto/ y no restrictivas en la Chile, $2016^{(10)}$ familia y personal de sanitario en la salud de los pacientes hospitalizados en unidades de cuidados intensivo.
Las visitas en las unidades de cuidados intensivos siguen siendo un tema de debate global quedando a criterio de cada institución sanitaria. Este estudio concluye que las visitas no restrictivas generan numerosos beneficios, favoreciendo la recuperación del paciente en lo físico y en lo emocional. Asimismo, en la familia se evidencia una mayor satisfacción con el personal y el sistema sanitario al tener un horario de visita flexible, siempre respetando y dando prioridad a las labores clínicas en el paciente. Como ya se indicó, el sistema de visitas en las unidades dependerá de los protocolos internos de cada hospital y de su cultura, por lo que es necesario gestar nueva evidencia para validar de forma concreta los beneficios de las familias en la recuperación de los usuarios críticamente enfermos. 
E.3 What factors affect implementation of early rehabilitation into intensive care unit practice? - A qualitative study with clinicians/

Australia, $2016^{(11) .}$
Identificar factores (barreras y facilitadores) que influyen en la implementación de la rehabilitación temprana de los pacientes hospitalizados en las unidades de cuidados intensivos.
E.4 Building Bridges between healthcare professionals, patients and families:

A coproduced and integrated approach to self-management support in stroke/ Londres, $2016^{\text {(12). }}$

E.5 Buscando humanizar los cuidados intensivos/ España, $2017{ }^{(13) .}$
Profundizar en la comprensión de cómo el apoyo de autogestión a la familia puede convertirse en área integral del cuidado diario y la rehabilitación.
Exponer los aspectos del proyecto $\mathrm{HU}-\mathrm{Cl}$ humanización desde el paciente, familia $y$ equipo de salud
La rehabilitación temprana aplicada a las personas hospitalizados en las unidades de cuidados intensivos tiene beneficios, los cuales conlleva a una recuperación segura, pronta y eficaz. El estudio expone factores que actúan de barreras y o facilitadores para la aplicación de la rehabilitación temprana, de los cuales destacan las expectativas del equipo de salud y el conocimiento previo que estos tienen, al igual que los factores del usuario. Ambos se unen en la importancia de transmitir a los familiares, la importancia de la rehabilitación como proceso vital en la recuperación, siendo la familia un elemento central que repercute en el pronóstico, motivación, sedación, delirio y la relación familiar. Los médicos reconocen la necesidad educativa hacia el equipo de salud como estrategia para nivelar los conocimientos y así reconocer a los familiares como un factor positivo para su recuperación. Por otro lado, se hace mención de las barreras como la falta de tiempo, personal y recursos, estos factores deben cambiar para un plan de mejora a futuro. Finalmente, se reconoce que la rehabilitación no es responsabilidad de una sola disciplina sino más bien de equipo multidisciplinario.

La autogestión familiar en la Unidad de Cuidados Intensivos ( $\mathrm{UCl})$, implica que los profesionales desarrollen habilidades y relaciones de apoyo con la familia de las personas críticamente enfermas para realizar prácticas eficientes que respondan a sus necesidades. Se ha demostrado que este tipo de enfoque ha mejorado los valores, que priorizan la asociación entre usuarios, familiares y profesionales. Existe la necesidad de implementar la autogestión para mejorar el compromiso y éxito con enfoques y métodos, más flexibles y abiertos.

Los avances tecnológicos en las UCls han crecido a pasos agigantados, pero al parecer ha dejado de lado lo más esencial, al usuario y su entorno. Contar una dinámica de trabajo organizada, con líneas estratégicas, actitud de compromiso y humanizada permite tener mayor conciencia ante la vulnerabilidad y necesidad de las personas críticamente enfermas y sus familias. Esto se obtiene a través del Proyecto $\mathrm{HU}-\mathrm{Cl}$ que abarca distintas áreas de trabajo, tales como, horarios de visita, comunicación, bienestar del paciente, participación familiar, entre otras.

Se puntualiza que la flexibilidad de horarios de visitas y la comunicación como aspectos beneficiosos para los pacientes, familias y personal de salud, siendo los más sencillo de implementar. Este punto se sustenta con la comunicación efectiva, la cuál es la principal necesidad expresada por la familia por la gran carga emocional asociada como el estrés, ansiedad y depresión, 
E.6 Evaluating the past to improve the future - A qualitative study of ICU patients' experiences/ Noruega, $2017^{(14)}$.
Investigar experimentaron recuperación rehabilitación durante su estadía y al alta los pacientes adultos hospitalizados en las unidades de cuidados intensivos.

permitiendo un ambiente de confianza y respeto al sentirse partícipe en los cuidados y en la toma de decisiones, dando así la posibilidad de contribuir en la recuperación de su familiar críticamente enfermo. Se hace imperativo la búsqueda de mayor evidencia para erradicar políticas arraigadas para distanciar a la familias, y por el contrario, acercarlas a las unidades y hacerlas participes de los cuidados para potenciar la recuperación del usuario.

cómo Los usuarios ingresados en la unidad de cuidados delirio. Describen alteraciones físicas y psicológicas durante su estadía. Todos los pacientes afrontaron la $\mathrm{UCl}$ de distintas maneras, algunos destacaron elementos que les permitieron hacer recordar como el diario y las fotografías que evidenciaban su estadía en la $\mathrm{UCl}$, mientras que otros preferían olvidar el período y seguir adelante. La entrega de información en folleto fue muy valorada por los usuarios y sus familias, ya que les ayudó a sobrellevar y entender que su experiencia vivida en esta unidad era normal. El estudio consideró que los sobrevivientes de la $\mathrm{UCl}$ y sus familias deben ser incluidos en los cuidados a través de entrega de material de información y programas de rehabilitación. Durante su recuperación, se destacó que los pacientes valoraron a los miembros de su familia como su principal fuente de información, sobre todo después de su hospitalización, ya que, con su experiencia de acompañamiento durante la hospitalización y relatos, estos entregaron tranquilidad y apoyo.

Existe escasa información con respecto a la incorporación de las familias en la rehabilitación/recuperación de los usuarios de cuidados intensivos. Se menciona la importancia de generar datos para ayudar a comprender mejor cómo las familias podrían apoyar la recuperación. Se destaca que, para empoderar a las familias en un marco de rehabilitación, se necesita estar abiertos y dispuestos de aprender de los usuarios y sus familias, ya que estos tienen potencial de conducir a la mejora de los resultados, por lo cual se debe realizar un cambio en el enfoque del personal de salud a un paradigma centrado en la familia. Se mencionan temas como la rehabilitación, la salud emocional, el apoyo, del compañerismo y la capacidad del personal para realizar esto. Se recomienda investigaciones futuras para explorar más esta área, ya que se deben considerar las barreras. 
E.8 Family in Rehabilitation, Empowering Careers for Improved

Malnutrition Outcomes: Protocol for the FREER Pilot Study/

Australia, 2019 (15).

E.9 Nurture-Empower-

Support: A HumanCentered

Approach to Understand and Support ICU Families/ USA, $2019^{(4)}$.
Determinar si la familia es importante en la rehabilitación y si su empoderamiento

presenta una mejora en la nutrición de los pacientes hospitalizados en unidades de cuidados intensivos.

Investigar cómo las intervenciones en las unidades de cuidados intensivos pueden facilitar el apoyo a las familias de pacientes hospitalizados.
La familia es un recurso poco utilizado para la recuperación del paciente crítico. La familia en la recuperación y empoderada (FREER), propone establecer una relación entre el cuidador principal y el equipo de salud a cargo para atender las demandas nutricionales, capacitándonos y entregándoles herramientas de cuidado a largo plazo, logrando empoderar al cuidador y darle compromiso.

Se fomenta desarrollar investigación atractiva, ya que podría llevar a cambios en las unidades de cuidados críticos.

Como resultados de este estudio emergen seis clases de necesidades que experimentan los familiares durante el proceso de hospitalización de una persona críticamente enferma, tales como necesidad de apoyo emocional continuo durante la hospitalización, mayor conciencia hacia los familiares por el equipo de salud, la búsqueda de construir relaciones con los profesionales, la necesidad de apoyo al equipo, la necesidad de ser incluidos y formar parte de la atención y la necesidad de cuidar de sí mismos. Por lo que se habla de NurtureEmpower-Support (NES), que describe cómo Nutrir, dando prioridad a proporcionar conocimientos al equipo y familia del cuidado del paciente, Autorizar, ofreciendo y otorgando ayuda suplementaria en base al estado de salud del usuario y Apoyar, a las familias para poder contribuir al equipo de salud y a la persona enferma.

Fuente: Tabla elaborada por las autoras.

Como segundo aspecto relevante, los estudios muestran que existen algunos factores o aspectos claves para fortalecer la recuperación del usuario críticamente enfermo. Autores describen elementos claves para la recuperación de las personas con alteraciones críticas de salud entre los que destacan: a) las expectativas y conocimiento médico incluyendo justificación de la rehabilitación, los beneficios percibidos y el impacto experimentado de los beneficios; b) la evidencia y la aplicación de rehabilitación, incluso cuándo intervenir; c) factores del paciente como el pronóstico, sedación, delirio, cooperación, motivación, objetivos y la presencia de la familia; d) consideraciones de seguridad, tales como estabilidad fisiológica del paciente y la presencia de dispositivos; e) influencias ambientales, como personal disponible, recursos, equipo, tiempo y prioridades en competencia; f) cultura y trabajo en equipo, participación del equipo 
multidisciplinario, roles profesionales, comunicación y cultura de la unidad, considerado el punto más relevante. ${ }^{(11)}$ Otros estudios reconocen a la familia como parte central y vital de la persona críticamente enferma. Sin embargo, el problema surge en la práctica clínica (en el día a día de la UCI), ya que las unidades de cuidados intensivos son áreas de alta complejidad, que se caracterizan por ser estresantes y generar una atmósfera emocionalmente lábil, tanto para profesionales como para pacientes y sus familias ${ }^{(1-3,12-13)}$.

Finalmente, la literatura revisada evidencia que se requiere de una gran autogestión de los equipos de salud y las familias, para que no sea arbitrario el ingreso y acceso ${ }^{(12)}$, se observan algunas metodologías nuevas, las cuales han sido aplicadas en países desarrollados, por tanto, la implementación de ellas en países en vías de desarrollo o con menor ingreso económico, aún no han sido implementadas ni reportadas para evaluar su eficacia. Por otra parte, se reconoce que, para empoderar a las familias en el marco de recuperación y rehabilitación, se requiere que el equipo de salud cohesionado, disponible y preparado para aprender de los usuarios y sus familias, ya que estos tienen potencial de conducir a la mejora de los resultados ${ }^{(11,16)}$ considerando las limitantes posibles que pueden presentarse y el tiempo que se debe tener para aplicar estas medidas. Se puntualiza que, para potenciar estas estrategias de trabajo, se deben realizar cambios con respecto al diseño, infraestructura y organización interna de las unidades de cuidados intensivos para así mejorar el bienestar y privacidad de los usuarios y sus familias, teniendo presente las demandas personales y emocionales de cada uno de los usuarios y sus familias ${ }^{(1)}$.

\section{Conclusiones}

La revisión realizada permite concluir que las unidades de paciente crítico continúan siendo un lugar restringido y de alta complejidad, por tanto, el ingreso de las familias es dificultoso. Si bien, los estudios revelan la importancia de incorporar a la familia en la recuperación del paciente crítico, ya que son reconocidos como un eje importante en este proceso de salud-enfermedad, aún faltan otros aspectos y áreas por investigar como las consecuencias en factores clínicos y hemodinámicos por mencionar algunas, 
para lograr demostrar de forma fehaciente por qué no se debe menospreciar el recurso familiar para la mejora de las personas con alteraciones críticas de salud.

De los resultados obtenidos en esta revisión, se destaca de forma indirecta el impacto positivo de la familia en la recuperación de la persona críticamente, sin embargo, aún existe un vacío de evidencia clínica esperada para la aplicación en las unidades de cuidados intensivos, debido alta exigencia de evidencia aplicable y demostrable que estas unidades requieren por su elevada complejidad.

Los estudios seleccionados hacen reflexionar sobre la necesidad de realizar intervenciones educativas al equipo de salud, como una estrategia para comprender la importancia de la recuperación y rehabilitación desde el ingreso del usuario a la unidad de cuidados intensivos, para así trasmitir estos conocimientos adquiridos por el equipo a la familia, con el objetivo de entregar mayor confianza y seguridad, fortaleciendo el cuidado. No se puede dejar de mencionar que existen diferentes mecanismos de ejecutar estas medidas de mejora, independientes cuál sea, todo lleva a que se evidencia un impacto significativo en los resultados y las experiencias de los familiares y en los pacientes de forma independientes. Finalmente se señala que es necesario desarrollar futuras investigaciones desde la disciplina de enfermería centrada en cuidados críticos, para así explorar desde la importancia de incorporar a las familias en la recuperación de la persona con alteraciones críticas de salud, que no tan solo tiene influencias en el ámbito emocional, sino también en ámbito clínico.

\section{Conflicto de intereses}

Los autores declaran no tener ningún conflicto de interés.

\section{Financiamiento}

Esta revisión de la literatura no conto con financiamiento para su ejecución. 


\section{Referencias Bibliográficas}

1. Escudero D, Vina L, Calleja C. Por una UCI de puertas abiertas, más confortable y humana. Es tiempo de cambio búsqueda aparte. Med Intensiva. 2014; 38(6):371-75.

2. Marquez-Herrera M, Carrillo-González G. La experiencia del familiar de la unidad de cuidados intensivos en Bucaramanga (Colombia): un estudio fenomenológico. Arch Med. 2015; 15 (1): 95-106.

3. Batista V, Coutinho L, Godoy F, De Freitas Góes H, Misue L, Silva S. Needs of the Relatives of Patients Hospitalized in an Intensive Therapy Unit. Rev Fund Care online. 2019; 11: 540-6.

4. Johnson J, Schmitz E, Ramnath V, Weibel N. Nurture-Empower-Support: A Human-Centered Approach to Understand and Support ICU Families. Pervasive Health'19: Proceedings of the 13th EAI International Conference on Pervasive Computing Technologies for Health care. 2019 [consultado 17 may 2020]; (SV): 119-28. Disponible en: https://doi.org/10.1145/3329189.3329247

5. Giannini A, Garrouste-Orgeas M, Latour, J. What's new in ICU visiting policies: can we continue to keep the doors closed? Intensive Care Med [Internet]. 2014 [Consultado 17 may 2020]; 40(5), 730-33. Disponible en: https://doi.org/10.1007/s00134-014-3267-y

6. Soares M, Silva U, Homena W, Fernandes G, De Moraes A, Brauer L, Lima M, De Marco F, Bozza F, Salluh J, ORCHESTRA (Organizational Characteristics in Critical Care) Study Investigators. Family care, visiting policies, ICU performance, and efficiency in resource use: insights from the ORCHESTRA study. Intensive Care Med. 2017; 4(43): 590-91.

7. Haines K. Engaging families in rehabilitation of people who are critically ill: an underutilized resource. Phys. Ther. [Internet]. 2018[Consultado 17 may 2020]; 9 (98):737-44. Disponible en: https://doi.org/10.1093/ptj/pzy066

8. Peñaherrera C, Soria J. Pregunta de investigación y estrategia PICOT. Rev. Med. FCM-UCSG. 2015; 19(1): 66-9.

9. Agard A, Egerod I, Tønnesen E, Lomborg K. From spouse to caregiver and back: a grounded theory study of post-intensive care unit spousal caregiving. J Adv Nurs. 2015; 71(8): 1892-903.

10. Anativia Montenegro P, Farias-Reyes D, Galiano-Gálvez M, Quiroga-Toledo N. Visita restrictiva / visita no restrictiva en una unidad de paciente crítico adulto. Aquichan. 2016; 16(3): 340-358. DOI: https://doi.org/10.5294/aqui.2016.16.3.6

11. Selina M, Remedios L, Denehy L, Knight L, Beach L, Granger C, et al. What factors affect implementation of early rehabilitation into intensive care unit practice? - A qualitative study with clinicians. J Crit Care. 2016;(38):137-43. 
12. Jones $F$, Pöstges $H$, Brimicombe L. Building Bridges between healthcare professionals, patients and families: A coproduced and integrated approach to self-management support in stroke. Neuro Rehabilitation [Internet]. 2016 [Consultado 17 may 2020]; 39: 471-80. Disponible en: https://doi.org/10.3233/NRE-161379

13. Heras G, Cruz M, Nin N. Seeking to humanize intensive care. Rev. bras. ter. intensiva [Internet]. 2017 [Consultado 2021 Feb 28]; 29 (1): 9-13. Disponible en: https://doi.org/10.5935/0103-507x.20170003

14. Olsen K, Nester M, Hansen B. Evaluating the past to improve the future - A qualitative study of ICU patients experiences. Intensive Crit Care Nurs. 2017; 43: 61-7.

15. Marshall S, Van der Meij B, Milte R, Collins $C$, de van der Schueren $M$, Banbury $M$, Warner $M$, Isenring E. Family in Rehabilitation, Empowering Carers for Improved Malnutrition Outcomes: Protocol for the FREER Pilot Study. JMIR Res Protoc [Internet]. 2019 [Consultado 17 may 2020] ;8(4): e12647. Disponible en: https://doi.org/10.2196/12647

16. Govindan S, Iwashyna T, Watson S, Hyzy R, Miller M. Issues of Survivorship are rarely addressed during Intensive Care Unit Stays. Ann Am Thorac Soc [Internet]. 2014 [Consultado 17 may 2020]; 4(11): 58791. Disponible en: https://doi.org/10.1513/AnnalsATS.201401-007BC

Cómo citar este artículo: Ceballos-Vásquez P, Campos-Fuentes M, González-Alegría V, Lobos-Lavín C. Impacto familiar en la recuperación de personas adultas críticamente enfermas: una revisión de la literatura. SANUS [Internet]. 2021[citado el $\underline{\mathrm{dd}} \mathrm{mm}$ aa]; 6: e201. Disponible en: DOI/link 